Original

\title{
Prevalencia de obesidad y obesidad abdominal en la población española de 65 y más años de edad: estudio ENPE
}

\author{
Carmen Pérez-Rodrigo ${ }^{\mathrm{a}, \mathrm{b}}$, Marta Gianzo Citores ${ }^{\mathrm{b}}$, Gotzone Hervás Bárbara ${ }^{\mathrm{b}}$ \\ y Javier Aranceta-Bartrina ${ }^{a, b, c, d, e, *}$ \\ a Departamento de Fisiología, Facultad de Medicina y Enfermería, Universidad del País Vasco (UPV/EHU), Leioa, Bizkaia, España \\ b Sociedad Española de Nutrición Comunitaria (SENC) \\ c Departamento de Ciencias de la Alimentación y Fisiología, Universidad de Navarra, Pamplona, Navarra, España \\ d Instituto Universitario de Investigaciones Biomédicas y Sanitarias (IUIBS), Universidad de Las Palmas de Gran Canaria, Las Palmas de Gran Canaria, España \\ e CIBEROBN, Biomedical Research Networking Center for Physiopathology of Obesity and Nutrition, Instituto de Salud Carlos III, Madrid, España
}

\section{INFORMACIÓN DEL ARTÍCULO}

\section{Historia del artículo:}

Recibido el 30 de agosto de 2020

Aceptado el 26 de octubre de 2020

On-line el xxx

\section{Palabras clave:}

Adultos mayores

Obesidad

Obesidad abdominal

Sarcopenia

Factores de riesgo

\section{R E S U M N}

Antecedentes y objetivos: Evaluar la prevalencia de obesidad y obesidad abdominal (OA) en población española $\geq 65$ años, analizar la influencia de algunos factores sociodemográficos y su asociación con factores de riesgo.

Métodos: La muestra procede del estudio ENPE, estudio transversal en muestra aleatoria representativa de la población no institucionalizada (2014-2015). Este análisis se refiere a población $\geq 65$ años $(n=1.233)$. Observadores entrenados realizaron mediciones antropométricas en los domicilios según protocolos internacionales estandarizados. Se consideró obesidad (índice de masa corporal [IMC] $\geq 30$ ); OA (cintura $>102 \mathrm{~cm}$ varones; $>88 \mathrm{~cm}$ mujeres).

Resultados: Se ha estimado una alta prevalencia de obesidad en adultos $\geq 65$ años, mayor en mujeres (40,1\% [IC95\% 36,4-43,8]) que en hombres (32,5\% [IC95\% 28,5-36,8]). La prevalencia de OA también es mayor en mujeres (69,9\% [IC95\% 66,4-73,1]) que en hombres (40,7\% [IC95\% 36,5-44,8]), y es aún mayor al definir OA considerando el índice cintura-cadera o el índice cintura-talla. El 39,8\% de tipificados como OA tienen IMC entre 25-29. Obesidad y OA son más frecuentes en el sur respecto al este, norte-este y centro y tienen relación inversa con el nivel educativo. Obesidad y OA se asocian con mayor probabilidad de hipercolesterolemia e hipertensión arterial. La obesidad sarcopénica también se asocia, además, con diabetes.

Conclusión: La prevalencia de obesidad y OA en adultos $\geq 65$ años es alta, mayor en mujeres, en personas con menor nivel educativo y en la región sur respecto al este, norte-este y centro. Es especialmente preocupante la alta prevalencia de OA por su asociación con complicaciones cardiovasculares, metabólicas y deterioro de la calidad de vida.

(C) 2020 Elsevier España, S.L.U. Todos los derechos reservados.

\section{Prevalence of obesity and abdominal obesity in Spanish population aged 65 years and over: ENPE study}

\section{A B S T R A C T}

Background and objectives: To assess the prevalence of obesity and abdominal obesity (AO) in the Spanish population aged $\geq 65$ years, to analyse the influence of selected sociodemographic factors and association with risk factors.

Methods: The sample comes from the ENPE study, a cross-sectional study of a representative sample of the non-institutionalized population (2014-2015). This analysis refers to the population $\geq 65$ years $(n=1,233)$. Trained observers performed anthropometric measurements at participants' homes following standard international protocols. Obesity was defined as body mass index (BMI) $\geq 30$; AO waist $>102 \mathrm{~cm}$ men; > $88 \mathrm{~cm}$ women.

\footnotetext{
* Autor para correspondencia.

Correos electrónicos: jaranceta@unav.es, javieraranceta@gmail.com (J. Aranceta-Bartrina).
} 
Results: Estimated prevalence of obesity in adults $\geq 65$ years was high, higher in women $(40.1 \%$ [95\% CI 36.4-43.8]) than in men (32.5\% [95\% CI 28.5-36.8]). The prevalence of AO was also higher in women (69.9\% [95\% CI 66.4-73.1]) than in men (40.7\% [95\% CI 36.5-44.8]), and estimates were even higher when defining $A O$ by waist-hip ratio or waist-height ratio. Of those classified as AO, 39.8\% have a BMI between 25-29. Obesity and AO are higher in the South region compared to East, North-East and Central regions and showed inverse association with educational level. Obesity and AO were associated with increased probability of hypercholesterolaemia and high blood pressure, sarcopenic obesity, and diabetes.

Conclusion: The prevalence of obesity and $\mathrm{AO}$ in adults aged $\geq 65$ years is high, higher in women, in people of lower educational level and in the South region compared to East, North-East and Central regions. The high prevalence of $\mathrm{OA}$ is especially worrying due to its association with cardiovascular and metabolic complications and poorer quality of life.

(c) 2020 Elsevier España, S.L.U. All rights reserved.

\section{Introducción}

La obesidad en adultos mayores contribuye a la aparición precoz de enfermedades crónicas y deficiencias funcionales, se asocia con peor calidad de vida y mortalidad ${ }^{1}$. Entre los factores biológicos, ambientales y sociales que contribuyen al aumento de peso, el envejecimiento es un determinante clave en la disminución de la tasa metabólica y actividad física ${ }^{2}$. Cada vez hay más personas mayores obesas debido al aumento de personas en edad avanzada, que se espera pase del 9,1\% al 15,9\% de la población mundial en $2050^{3}$.

Entre 1975 y 2014 la prevalencia de obesidad en el mundo, en adultos, se ha triplicado en hombres y se ha duplicado en mujeres ${ }^{3}$. Actualmente en Europa se estima que una de cada 5 personas mayores de 60 años padece obesidad y el $42 \%$ sobrepeso $^{4}$. De acuerdo con el proyecto SHARE, en Europa, entre 2005 y 2013 aumentó la prevalencia de sobrepeso en hombres mayores de 60 años y en mujeres mayores de 80 años, y la obesidad, en hombres y mujeres a partir de 60 años $^{5}$. Esta tendencia también se observa en España a partir del análisis de las Encuestas de Salud (1987-2012) ${ }^{6}$.

La alta prevalencia de obesidad supone un reto para la atención sanitaria y un importante problema de salud pública por el elevado coste sanitario añadido, consecuencia del mayor riesgo de aparición de comorbilidades y enfermedades crónicas relacionadas con la obesidad, como diabetes, hipertensión, enfermedades cardiovasculares, ciertos tipos de cáncer o alteraciones articulares, entre otras ${ }^{1,3}$. Proyecciones recientes en España estiman un incremento del $16 \%$ en el número de personas afectadas por exceso de peso para 2030 , lo que implicaría un aumento del $58 \%$ en sobrecoste sanitario directo ${ }^{7}$.

Desde hace años la tipificación ponderal en los adultos mayores se clasifica a partir del índice de masa corporal (IMC), utilizando los mismos puntos de corte que en adultos más jóvenes, IMC $\geq 25$ para sobrepeso e IMC $\geq 30$ para obesidad. Los análisis poblacionales de la asociación entre IMC y mortalidad total y por diferentes causas concluyen que el valor asociado con menor riesgo de mortalidad aumenta con la edad, desde $22 \mathrm{~kg} / \mathrm{m}^{2}$ entre 35 y 49 años, $23 \mathrm{~kg} / \mathrm{m}^{2}$ entre los 50 y 69 años y $24-25 \mathrm{~kg} / \mathrm{m}^{2}$ a partir de 70 años ${ }^{8}$.

La relación entre IMC y morbimortalidad asociada puede estar afectada por la distribución de la grasa corporal. Para una mejor caracterización del exceso ponderal y evaluación del riesgo asociado, además del IMC, se recomienda utilizar indicadores de distribución de la grasa como el perímetro de la cintura $(\mathrm{PC})^{9}$, o el índice cintura-talla $(\mathrm{C}-\mathrm{T})^{10}$, también en adultos mayores $^{11}$.

La sarcopenia, pérdida primaria de masa muscular y fuerza o función física muscular, es un problema vinculado con el envejecimiento $^{12}$. La asociación de obesidad y sarcopenia, denominada obesidad sarcopénica (OS), aumenta las comorbilidades en los adultos mayores ${ }^{11}$. El perímetro de la pantorrilla (PP) es un indicador sensible de la masa muscular en los ancianos. Se ha propuesto como punto de corte valores $<31 \mathrm{~cm}^{12}$.

En este contexto, el objetivo de este estudio es evaluar la prevalencia de obesidad y obesidad abdominal (OA) con base en mediciones antropométricas individuales, según diferentes criterios, en población española de 65 y más años, analizar su distribución según la edad, el sexo, ubicación geográfica, la influencia de algunos factores sociodemográficos y su asociación con factores de riesgo cardiovascular.

\section{Métodos}

Este estudio es parte del estudio ENPE (Estudio Nutricional de la Población Española), estudio observacional, transversal, diseñado con el fin de obtener una actualización sobre los hábitos de consumo alimentario, datos antropométricos y actividad física en población española mayor de 3 años no institucionalizada. El estudio se realizó en una muestra probabilística de base poblacional, seleccionada por un procedimiento aleatorio polietápico, descrito en publicaciones precedentes $^{13}$, con estratificación de las unidades de primera etapa (secciones censales) por comunidades autónomas. Las unidades de segunda etapa fueron las viviendas principales, y en cada hogar se seleccionaba un individuo con cuotas controladas y afijación proporcional según densidad poblacional por grupos de edad (3-8 años, 9-18 años, 19-64 años, $\geq 65$ años), sexo y tamaño del municipio (ámbito poblacional: personas residentes en viviendas familiares principales de todo el territorio nacional). Se excluyó de las mediciones antropométricas personas que no podían mantenerse en bipedestación o con brazos y/o piernas inmovilizados con férulas de escayola que no podían retirarse. Se reclutó la muestra por rutas aleatorias, puerta a puerta. La recogida de datos se extendió durante un año (mayo 2014-mayo 2015). Se realizó mediante entrevista personal en el domicilio del participante. La muestra final incluyó 6.800 individuos, $1.233 \geq 65$ años.

\section{Mediciones antropométricas}

Realizaron las mediciones antropométricas individuales encuestadores profesionales bien entrenados, que recibieron formación específica de manera presencial de los autores del estudio, siguiendo procedimientos internacionales estandarizados ${ }^{14}$ probados previamente en un estudio piloto. Las mediciones se realizaron con los sujetos en bipedestación, descalzos y con ropa ligera: Talla, medida en centímetros utilizando un tallímetro portátil Seca modelo 213 (Seca GmbH \& Co. Kg.; Hamburgo, Alemania) (límites, $0-200 \mathrm{~cm}$; precisión, $0,1 \mathrm{~cm}$ ). Peso corporal, utilizando básculas digitales calibradas Seca modelo 803 (límites, 0,1-150 kg; precisión, 0,1 kg). PC, medido en el punto medio entre el último reborde costal y la cresta iliaca, a la altura de la mitad de la axila, perpendicular al eje longitudinal del tronco, al final 
de una espiración normal, con los brazos relajados a cada lado. Perímetro de la cadera, medido en el punto de máxima circunferencia sobre las nalgas en un plano horizontal perpendicular al eje longitudinal del tronco. Se calculó el IMC como el peso $(\mathrm{kg}) /$ talla $(\mathrm{m})^{2}$; el índice cintura-cadera $(\mathrm{C}-\mathrm{C})$ como $\mathrm{PC}(\mathrm{cm}) /$ perímetro de la cadera $(\mathrm{cm})$ y el C-T como PC $(\mathrm{cm}) /$ talla $(\mathrm{cm})$ y el índice de adiposidad corporal ${ }^{13}$ como [perímetro de la cadera $(\mathrm{cm}) /($ talla $\left.(m)^{1,5}\right)-18$. Se midió el PP en la pierna derecha, en bipedestación en el punto de máxima circunferencia, clasificando valores $<31 \mathrm{~cm}$ como sarcopenia. Todos los perímetros se midieron por triplicado con una cinta métrica inextensible modelo Seca 201 (intervalo, $0-150 \mathrm{~cm}$; precisión, 0,1 cm).

Los controles de calidad permitieron estimar errores técnicos de medición relativos intraobservador e interobservadores $<1 \%$ para todos los parámetros antropométricos referidos, inferiores a los considerados aceptables ${ }^{14}$.

Se clasificó como sobrepeso valores de IMC $\geq 25$ y como obesidad, IMC $\geq 30$. La OA se definió como valores de PC $>102 \mathrm{~cm}$ en varones y $>88 \mathrm{~cm}$ en mujeres, según los criterios de la $\mathrm{OMS}^{9}$. Según el C-C, se definió OA como valores $>0,90$ en varones $\mathrm{y}>0,85$ en mujeres $^{9}$. Considerando el C-T, se definió OA para valores $>0,55^{13}$.

\section{Factores sociodemográficos, estilos de vida y problemas de salud}

Se ha considerado sexo, grupos de edad (65-74 años; $\geq 75$ años); nivel educativo (menos de estudios primarios, estudios primarios, secundarios y superiores); nivel socioeconómico familiar según nivel de estudios y ocupación (alto-medio alto; medio-medio; medio-bajo; bajo); región geográfica (norte-oeste [Galicia, Asturias], norte-este [Cantabria, País Vasco], centro [Castilla y León, Madrid, Extremadura, Castilla-La Mancha], centro-este [Navarra, La Rioja, Aragón], este [Cataluña, Baleares, Comunidad Valenciana] y sur [Canarias, Andalucía, Murcia]) y tamaño de localidad de residencia desde menos de 5.000 hasta más de 200.000 habitantes.

También se ha considerado el hábito de fumar (fumador, exfumador, no fumador) y el nivel de actividad física (baja, moderada, alta), estimado mediante el cuestionario validado IPAQ ${ }^{15}$. La presencia de problemas de salud como hipercolesterolemia, hipertensión arterial (HTA) y diabetes se valoró como información reportada por los encuestados, mediante la pregunta: ¿Padece de alguno de los siguientes problemas crónicos de salud?: colesterol alto, tensión arterial alta y diabetes con opciones de respuesta sí/no.

\section{Análisis de los datos}

El análisis se realizó en los individuos $\geq 65$ años con información completa para las variables de interés (1.176 de IMC [95,4\%], 1.185 de PC y cadera [96,1\%] y 960 [77,8\%] para PP). Se calculó la prevalencia de obesidad, OA y OS con su intervalo de confianza del 95\% (IC95\%) por sexo y grupo de edad. Se ponderó la muestra de acuerdo con la distribución de la población española. Se utilizó la prueba t de Student para comparar medias por sexo y grupo de edad. Se utilizaron la prueba $\chi^{2}$ y el z-test para comparar las tasas de prevalencia según edad y sexo. Se ha analizado la asociación de factores sociodemográficos con la prevalencia de obesidad y OA mediante modelos de regresión logística univariados y modelos ajustados por edad y sexo. También se ha analizado mediante modelos de regresión logística la asociación de la obesidad, OA y OS con factores de riesgo cardiovascular como hipercolesterolemia, HTA y diabetes, ajustados por edad, sexo, nivel educativo (menos que primarios, primarios, secundarios, superiores), consumo de tabaco (no, exfumador, fumador), y actividad física (baja, moderada, alta). El análisis tuvo en cuenta el diseño complejo de la muestra. Se llevó a cabo utilizando el paquete IBM SPSS v. 24.0.

\section{Consideraciones éticas}

Se informó a todos los participantes de los objetivos y procedimientos del estudio; dieron por escrito su consentimiento para participar. El protocolo final obtuvo la aprobación del Comité Ético de Investigación Clínica de Euskadi (PI2015147) y se llevó a cabo de acuerdo con la Declaración de Helsinki para estudios en humanos de la Asociación Médica Mundial, con estricto respeto a la confidencialidad de la información de acuerdo con la Ley Orgánica 15/1999, de 13 de diciembre, de protección de datos de carácter personal en todos los procesos de grabación y tratamiento de la información obtenida.

\section{Resultados}

En la tabla 1 se describe la distribución del colectivo estudiado y en la tabla 2, las características antropométricas por grupos de edad y sexo. En el grupo de edad 65-74 años se observaron diferencias significativas por sexo en todos los indicadores antropométricos considerados, salvo en PP. En el grupo de 75 y más años se observaron diferencias significativas por sexo para peso, talla, $\mathrm{PC}$, perímetro de cadera, $\mathrm{C}-\mathrm{C}$ e índice de adiposidad corporal.

Con la edad disminuyen significativamente el peso y la talla, tanto en hombres como en mujeres, pero no varía significativamente el IMC. También disminuye significativamente el perímetro de cadera, índice C-T e índice de adiposidad corporal en hombres.

\section{Obesidad y obesidad abdominal}

La prevalencia de obesidad (IMC $\geq 30$ ) 40,1\% (IC95\% 36,4-43,8), valores de IMC $\geq 35,14,2 \%$ (IC95\% 11,7-16,9), y OA, que alcanza el $69,9 \%$ (IC95\% 66,4-73,1) según el PC y tasas superiores al considerar como criterio el índice $\mathrm{C}-\mathrm{C}$ y el índice $\mathrm{C}-\mathrm{T}$, así como la coincidencia de obesidad y OA (PC) 37,0\% (IC95\% 33,4-40,6) son mayores en mujeres que en hombres (tabla 3 ).

El 43\% de los clasificados como OA según el índice C-T no se clasifican como OA según el PC. El 39,8\% de los clasificados como OA (PC) tienen IMC entre 25 y $29 \mathrm{~kg} / \mathrm{m}^{2}$.

No se observan diferencias significativas entre los dos grupos de edad considerados. En contraste, el 3,7\% (IC95\% 2,5-5,3) de las mujeres presentan valores del IMC $<21,5,3 \%$ (IC95\% 3,0-8,2) entre las de $\geq 75$ años.

\section{Obesidad y sarcopenia}

La prevalencia de sarcopenia se estima en el 17,7\% (IC95\% $14,3-21,7$ ) en hombres y $21,9 \%$ (IC95\% 18,7-25,5) en mujeres. La prevalencia de OS 10,9\% (IC95\% 8,7-13,3) y la coincidencia de OA y sarcopenia $22,0 \%$ (IC95\% 19,1-25,1) son más elevadas en mujeres. En los hombres, OS y OA sarcopénica son significativamente mayores en el grupo de edad entre 65 y 74 años.

\section{Factores sociodemográficos, obesidad y obesidad abdominal}

En la tabla 4 se muestran las odds ratio (OR) de prevalencia crudas del análisis univariado de la asociación de factores sociodemográficos con la prevalencia de obesidad y OA. La figura $1 \mathrm{~A}, \mathrm{~B}$ muestra las OR de prevalencia ajustadas por edad y sexo para los factores sociodemográficos considerados como variables independientes en modelos con obesidad (fig. 1A) y OA (fig. 1B) como variables dependientes.

Ajustado por edad, la probabilidad de obesidad es menor en hombres (OR 0,72; IC95\% 0,56-0,92); disminuye progresivamente a mayor nivel educativo respecto a quienes no han completado estudios primarios, y en cuanto a la región geográfica, la probabilidad es 
Tabla 1

Descripción de las características sociodemográficas del colectivo muestral

\begin{tabular}{|c|c|c|c|}
\hline Características & $\begin{array}{l}\text { Total } \\
\mathrm{n}(\%)\end{array}$ & $\begin{array}{l}\text { Hombres } \\
\mathrm{n}(\%)\end{array}$ & $\begin{array}{l}\text { Mujeres } \\
\mathrm{n}(\%)\end{array}$ \\
\hline Sujetos & 1.233 & 533 & 700 \\
\hline \multicolumn{4}{|l|}{ Grupos de edad } \\
\hline 65 a 74 años & $754(61,2)$ & $336(63,0)$ & $418(59,7)$ \\
\hline$\geq 75$ años & $479(38,8)$ & $197(37,0)$ & $282(40,3)$ \\
\hline \multicolumn{4}{|l|}{ Región geográfica } \\
\hline Norte-oeste & $189(15,3)$ & $77(14,5)$ & $112(16,0)$ \\
\hline Norte-este & $147(11,9)$ & $64(12,0)$ & $83(11,9)$ \\
\hline Centro & $299(24,2)$ & $138(25,9)$ & $161(23,0)$ \\
\hline Centro-este & $224(18,2)$ & $96(18,0)$ & $128(18,3)$ \\
\hline Este & $198(16,1)$ & $88(16,5)$ & $110(15,7)$ \\
\hline Sur & $176(14,3)$ & $70(13,1)$ & $106(15,1)$ \\
\hline \multicolumn{4}{|l|}{ Nivel educativo } \\
\hline Menos que primarios & $522(45,6)$ & $188(37,8)$ & $334(51,5)$ \\
\hline Primarios & $403(35,2)$ & $181(36,4)$ & $222(34,3)$ \\
\hline Secundarios & $127(11,1)$ & $76(15,3)$ & $51(7,9)$ \\
\hline Superiores & $93(8,1)$ & $52(10,5)$ & $41(6,3)$ \\
\hline \multicolumn{4}{|l|}{ Tamaño del hábitat } \\
\hline Menos de 5.000 habitantes & $212(17,2)$ & $95(17,8)$ & $117(16,7)$ \\
\hline Entre 5.000 y 15.000 habitantes & $249(20,2)$ & $107(20,1)$ & $142(20,3)$ \\
\hline Entre 15.000 y 50.000 habitantes & $221(17,9)$ & $96(18,0)$ & $125(17,9)$ \\
\hline Entre 50.000 y 200.000 habitantes & $274(22,2)$ & $124(23,3)$ & $150(21,4)$ \\
\hline Más de 200.000 habitantes & $277(22,5)$ & $111(20,8)$ & $166(23,7)$ \\
\hline
\end{tabular}

Tabla 2

Descripción de las características antropométricas del colectivo muestral

\begin{tabular}{|c|c|c|c|c|c|c|c|c|c|}
\hline & \multicolumn{3}{|c|}{ Hombres } & \multirow[t]{2}{*}{$\mathrm{p}$ (edad) } & \multicolumn{3}{|c|}{ Mujeres } & \multirow[t]{2}{*}{$\mathrm{p}$ (edad) } & \multirow[t]{2}{*}{$\mathrm{p}(\operatorname{sexo})$} \\
\hline & $\mathrm{n}$ & Media (IC95\% Li-Ls) & $\mathrm{DE}$ & & $\mathrm{n}$ & Media (IC95\% Li-Ls) & $\mathrm{DE}$ & & \\
\hline \multicolumn{10}{|l|}{65 a 74 años } \\
\hline Edad (años) & 336 & $69(68-69)$ & 69 & & 418 & $69(68-69)$ & 3 & & \\
\hline Peso $(\mathrm{kg})$ & 328 & $80,9(79,5-82,2)$ & 12,6 & 0,007 & 404 & $71,8(70,5-73,1)$ & 13,1 & 0,032 & $<0,001$ \\
\hline Talla $(\mathrm{cm})$ & 324 & $168,8(168,1-169,5)$ & 6,6 & $<0,001$ & 401 & $156,9(156,2-157,6)$ & 6,9 & 0,001 & $<0,001$ \\
\hline Índice de masa corporal $\left(\mathrm{kg} / \mathrm{m}^{2}\right)$ & 323 & $28,4(27,9-28,8)$ & 3,9 & & 397 & $29,2(28,7-29,7)$ & 5,3 & & 0,049 \\
\hline Circunferencia de la cintura $(\mathrm{cm})$ & 324 & $100,1(98,9-101,3)$ & 10,9 & & 407 & $95,0(93,7-96,3)$ & 13,9 & & $<0,001$ \\
\hline Circunferencia de la cadera $(\mathrm{cm})$ & 324 & $102,3(101,3-103,3)$ & 9,6 & 0,011 & 407 & $106,4(105,1-107,6)$ & 13,3 & & $<0,001$ \\
\hline Índice cintura-cadera & 324 & $0,98(0,97-0,99)$ & 0,09 & & 407 & $0,89(0,89-0,90)$ & 0,09 & & $<0,001$ \\
\hline Índice cintura-talla & 324 & $0,59(0,59-0,60)$ & 0,07 & 0,007 & 401 & $0,61(0,60-0,62)$ & 0,09 & & 0,014 \\
\hline Índice adiposidad corporal & 324 & $28,7(28,2-29,2)$ & 4,7 & $<0,001$ & 401 & $36,3(35,6-37,1)$ & 7,6 & & $<0,001$ \\
\hline Circunferencia de la pantorrilla $(\mathrm{cm})$ & 240 & $35,3(34,4-36,3)$ & 7,4 & & 314 & $35,0(34,4-35,7)$ & 6,0 & & \\
\hline \multicolumn{10}{|l|}{75 y más años } \\
\hline Edad (años) & 197 & $81(81-82)$ & 4 & & 282 & $81(81-82)$ & 4 & & \\
\hline Peso $(\mathrm{kg})$ & 191 & $77,0(75,3-78,7)$ & 11,0 & & 269 & $69,6(68,2-71,1)$ & 12,7 & & $<0,001$ \\
\hline Talla $(\mathrm{cm})$ & 191 & $165,7(164,6-166,9)$ & 7,6 & & 266 & $154,9(154,0-155,9)$ & 8,0 & & $<0,001$ \\
\hline Índice de masa corporal $\left(\mathrm{kg} / \mathrm{m}^{2}\right)$ & 190 & $28,1(27,5-28,6)$ & 3,8 & & 266 & $29,1(28,5-29,8)$ & 5,4 & & 0,017 \\
\hline Circunferencia de la cintura $(\mathrm{cm})$ & 188 & $101,6(99,6-103,5)$ & 12,9 & & 266 & $95,9(94,4-97,3)$ & 12,4 & & $<0,001$ \\
\hline Circunferencia de la cadera $(\mathrm{cm})$ & 188 & $105,0(103,2-106,9)$ & 12,1 & & 266 & $106,0(104,6-107,4)$ & 11,9 & & 0,023 \\
\hline Índice cintura-cadera & 188 & $0,97(0,95-0,99)$ & 0,10 & & 266 & $0,90(0,90-0,91)$ & 0,08 & & $<0,001$ \\
\hline Índice cintura-talla & 188 & $0,61(0,60-0,63)$ & 0,08 & & 266 & $0,62(0,61-0,63)$ & 0,09 & & \\
\hline Índice adiposidad corporal & 188 & $31,4(30,4-32,2)$ & 6,3 & & 266 & $37,1(36,2-37,9)$ & 7,5 & & $<0,001$ \\
\hline Circunferencia de la pantorrilla $(\mathrm{cm})$ & 167 & $35,7(34,8-36,6)$ & 5,6 & & 239 & $35,4(34,4-36,4)$ & 7,8 & & \\
\hline
\end{tabular}

DE: desviación estándar; IC: intervalo de confianza; Li: límite inferior IC95\%; Ls: límite superior IC95\%; p: nivel de significación t de Student comparación medias.

menor en las regiones norte-este, este y centro respecto a la región sur.

Por lo que se refiere a la $\mathrm{OA}$, ajustado por edad, también es menor la probabilidad en hombres, OR 0,30 (IC95\% 0,24-0,38) (fig. 1B) y se asocia de forma inversa con el nivel de estudios. La probabilidad de OA es menor en las regiones norte-este, este y centro respecto a la región sur y es mayor en localidades de menos de 5.000 habitantes respecto a las de mayor tamaño.

\section{Obesidad, obesidad abdominal y factores de riesgo cardiovascular}

En la figura $2 \mathrm{~A}-\mathrm{C}$ se muestran las $\mathrm{OR}$ de prevalencia ajustadas por edad, sexo, nivel educativo, hábito tabáquico y nivel de actividad física asociadas a la obesidad, OA (PC), OA (índice C-T) y OS en relación con la presencia de hipercolesterolemia, HTA o diabetes. Obesidad, OA, según PC y según índice C-T, y OS se asocian con mayor probabilidad de hipercolesterolemia e HTA. La OS aumenta la probabilidad de diabetes, OR 2,2 (IC95\% 1,3-3,7).

\section{Discusión}

En el estudio ENPE se ha estimado una alta prevalencia de obesidad en adultos de 65 y más años de edad, mayor en mujeres $(40,1 \%)$ que en hombres (32,5\%). La prevalencia de OA según el PC también es mayor en mujeres $(69,9 \%)$ que en hombres $(40,7 \%)$, y cuando se utilizan otros criterios de OA, como el índice C-C o el índice C-T, las estimaciones son aún más elevadas. Estos datos sugieren que una proporción considerable de personas clasificadas en función 
Tabla 3

Tipificación ponderal, obesidad abdominal según distintos criterios, sarcopenia y coincidencia de las tres condiciones por grupos de edad y sexo

\begin{tabular}{|c|c|c|c|c|}
\hline & \multicolumn{2}{|c|}{ Hombres } & \multicolumn{2}{|c|}{ Mujeres } \\
\hline & $\mathrm{n}$ & \% (IC95\% Li-Ls) & $\mathrm{n}$ & \% (IC95\% Li-Ls) \\
\hline \multicolumn{5}{|l|}{ Todos } \\
\hline IMC $<25$ & 97 & $19,6(16,2-23,2)$ & 140 & $20,8(17,8-24,0)$ \\
\hline IMC 25-29,9 & 236 & $48,0(43,6-52,4)$ & 264 & $39,1(35,4-42,7)$ \\
\hline $\mathrm{IMC} \geq 30$ & 160 & $32,4(28,5-36,8)$ & 270 & $40,1(36,4-43,8)^{* *}$ \\
\hline $\mathrm{IMC} \geq 35$ & 18 & $3,6(2,2-5,5)$ & 96 & $14,2(11,7-16,9)^{*}$ \\
\hline Obesidad abdominal PC $(\mathrm{H}>102 \mathrm{~cm} ; \mathrm{M}>88 \mathrm{~cm})$ & 219 & $40,7(36,5-44,8)$ & 505 & $69,9(66,4-73,1)^{* *}$ \\
\hline Obesidad abdominal $\mathrm{C}-\mathrm{C}(\mathrm{H}>0,90 ; \mathrm{M}>0,85)$ & 430 & $85,9(82,6-88,7)$ & 528 & $79,5(76,2-82,3)^{* *}$ \\
\hline Obesidad abdominal C-T $(\geq 0,55)$ & 387 & $71,8(67,9-75,5)$ & 530 & $73,3(70,0-76,4)$ \\
\hline Obesidad + obesidad abdominal PC & 115 & $23,3(19,8-27,3)$ & 249 & $37,0(33,4-40,6)^{* *}$ \\
\hline Sarcopenia & 72 & $17,7(14,3-21,7)$ & 124 & $21,9(18,7-25,5)$ \\
\hline Obesidad + sarcopenia & 51 & $10,3(7,9-13,3)$ & 73 & $10,9(8,7-13,3)$ \\
\hline Obesidad abdominal PC + sarcopenia & 63 & $11,7(9,2-14,6)$ & 159 & $22,0(19,1-25,1)^{* *}$ \\
\hline Obesidad + OB abdominal PC + sarcopenia & 51 & $10,3(7,9-13,3)$ & 73 & $10,9(8,7-13,3)$ \\
\hline \multicolumn{5}{|l|}{65 a 74 años } \\
\hline IMC $<25$ & 61 & $18,7(14,9-23,4)$ & 85 & $21,3(17,4-25,4)$ \\
\hline IMC 25-29,9 & 159 & $49,2(43,7-54,5)$ & 159 & $39,6(35,0-44,5)$ \\
\hline $\mathrm{IMC} \geq 30$ & 104 & $32,1(27,2-37,3)$ & 157 & $39,1(34,5-44,0)$ \\
\hline $\mathrm{IMC} \geq 35$ & 14 & $4,3(2,5-6,8)$ & 50 & $12,4(9,4-15,8)$ \\
\hline Obesidad abdominal PC $(\mathrm{H}>102 \mathrm{~cm} ; \mathrm{M}>88 \mathrm{~cm})$ & 143 & $39,7(34,8-44,8)$ & 297 & $68,9(64,4-73,1)$ \\
\hline Obesidad abdominal $\mathrm{C}-\mathrm{C}(\mathrm{H}>0,90 ; \mathrm{M}>0,85)$ & 288 & $85,8(81,7-89,1)$ & 318 & $78,1(73,9-81,9)$ \\
\hline Obesidad abdominal C-T $(\geq 0,55)$ & 250 & $69,6(64,5-74,0)$ & 308 & $71,5(67,1-75,6)$ \\
\hline Obesidad + obesidad abdominal PC & 72 & $22,1(18,0-27,0)$ & 146 & $36,4(31,8-41,2)$ \\
\hline Sarcopenia & 48 & $19,1(14,6-24,2)$ & 63 & $19,5(15,5-24,2)$ \\
\hline Obesidad + sarcopenia & 41 & $12,5(9,4-16,6)^{*}$ & 43 & $10,8(8,0-14,0)$ \\
\hline Obesidad abdominal PC + sarcopenia & 52 & $14,5(11,1-18,4)^{*}$ & 104 & $24,2(20,3-28,3)^{* *}$ \\
\hline Obesidad + OB abdominal PC + sarcopenia & 41 & $12,5(9,4-16,6)^{*}$ & 43 & $10,8(8,0-14,0)$ \\
\hline \multicolumn{5}{|l|}{75 y más años } \\
\hline IMC $<25$ & 36 & $21,3(15,7-28,1)$ & 55 & $20,1(15,7-25,2)$ \\
\hline IMC 25-29,9 & 77 & $45,6(38,4-53,4)$ & 105 & $38,4(32,8-44,3)$ \\
\hline $\mathrm{IMC} \geq 30$ & 56 & $33,1(26,5-40,7)$ & 113 & $41,5(35,7-47,3)$ \\
\hline $\mathrm{IMC} \geq 35$ & 4 & $2,3(0,8-5,5)$ & 46 & $16,8(12,7-21,6)$ \\
\hline Obesidad abdominal PC $(\mathrm{H}>102 \mathrm{~cm} ; \mathrm{M}>88 \mathrm{~cm})$ & 76 & $42,5(35,2-49,5)$ & 208 & $71,3(65,9-76,2)$ \\
\hline Obesidad abdominal $\mathrm{C}-\mathrm{C}(\mathrm{H}>0,90 ; \mathrm{M}>0,85)$ & 142 & $85,9(80,2-90,7)$ & 211 & $81,6(76,7-86,1)$ \\
\hline Obesidad abdominal C-T $(\geq 0,55)$ & 137 & $76,3(69,5-81,9)$ & 221 & $75,9(70,5-80,3)$ \\
\hline Obesidad + obesidad abdominal PC & 43 & $25,5(19,5-32,6)$ & 103 & $37,9(32,1-43,6)$ \\
\hline Sarcopenia & 24 & $15,4(10,6-22,1)$ & 61 & $25,1(20,0-30,8)$ \\
\hline Obesidad + sarcopenia & 10 & $6,1(3,1-10,3)$ & 13 & $10,9(7,7-15,1)$ \\
\hline Obesidad abdominal PC + sarcopenia & 11 & $6,0(3,3-10,3)$ & 55 & $18,8(14,7-23,6)$ \\
\hline Obesidad + OB abdominal PC + sarcopenia & 10 & $6,1(3,1-10,3)$ & 30 & $10,9(7,7-15,1)$ \\
\hline
\end{tabular}

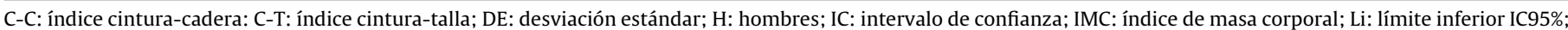

Ls: límite superior IC95\%; M: mujeres; OB: obesidad; p: nivel de significación test de $\chi^{2}$; PC: perímetro de la cintura; Sarcopenia: perímetro de pantorrilla < 31 cm.

$\mathrm{p}<0,05$.

$\mathrm{p}<0,01$.

del IMC como no obesas se clasifican como OA, condición asociada con mayor riesgo metabólico y cardiovascular.

Con los procesos de envejecimiento se producen cambios en la composición corporal, con aumento de la masa grasa y modificaciones en su distribución, con tendencia a mayor depósito de grasa intraabdominal sobre todo en mujeres, menor masa magra y disminución de la masa ósea. En este grupo de edad con frecuencia es problemático estimar la talla por alteraciones en los discos intervertebrales, cifoescoliosis y dificultades para mantenerse en bipedestación con rodillas y caderas extendidas ${ }^{16}$. El IMC no diferencia el componente muscular del adiposo. A pesar de ello, se utiliza el IMC y los mismos puntos de corte que en población adulta para estimar la prevalencia de sobrepeso y obesidad en ancianos, aunque se recomienda añadir el PC a este indicador ${ }^{16}$. Se ha sugerido que el índice $C$-T podría estimar mejor el riesgo asociado con el exceso de adiposidad corporal ${ }^{10,11}$.

Publicaciones recientes sitúan las tasas de prevalencia de obesidad en población mayor de 50 años en España (datos autorreferidos) entre las más altas de Europa, aunque con tendencia decreciente $^{6}$. A partir de mediciones individuales, en EE. UU. (datos NHANES 2013-2014), se ha estimado una prevalencia de obesidad del 38,5\% en mayores de 60 años ${ }^{17}$ y en México (datos ENSANUT 2012) se estimaba que el 30,2\% de los mayores de 60 años eran obesos $^{18}$. Los resultados del National Food, Nutrition and Physical Activity Survey $2015-2016$ en Portugal ${ }^{19}$ reflejan una prevalencia de obesidad del 39,2\% (65-84 años), mayor que en ENPE (36,9\%) ( $\geq 65$ años).

Las tasas estimadas en ENPE son similares a las referidas para este grupo de edad en ENRICA (2008-2010) ${ }^{20}$ (35\%) y en mujeres en EXERNET (2008-2009) ${ }^{21}$ (40,9\%), aunque son mayores en hombres (32,5\%) que en EXERNET (26,6\%). Incluso, análisis previos como PREV-ICTUS (2005) ${ }^{22}$ o DORICA (2004) ${ }^{23}$ expresaban prevalencias en torno al 35\%. En todos los casos, la prevalencia de obesidad es mayor en mujeres que en hombres, en la misma línea que los resultados del ENPE. Esta distribución asimétrica entre sexos podría deberse, por una parte, a que los hombres tienen mayor cantidad relativa de masa magra que las mujeres, y por otra, a que las mujeres tienen mayor tendencia a acumular grasa ${ }^{16}$.

Analizando conjuntamente estos datos, se observa que en las últimas décadas se ha producido un leve aumento de la sobrecarga ponderal, que podría deberse al progresivo aumento de esperanza de vida ${ }^{24}$, y quizá también a modificaciones en los estilos de vida en la población adulta mayor. Es más, de acuerdo con las proyecciones del avance del problema en España se estima que en la próxima década avanzará de manera que supondrá un sobrecoste en torno al $58 \%$ en gasto sanitario directo ${ }^{7}$. A ello debemos añadir que, en el 
Tabla 4

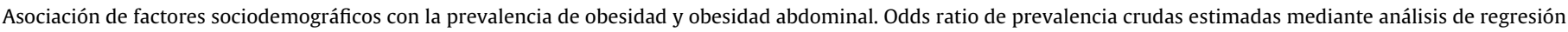
logística

\begin{tabular}{|c|c|c|c|c|}
\hline & $\begin{array}{l}\text { Obesidad } \\
\text { OR (IC95\% Li-Ls) }\end{array}$ & $\mathrm{p}$ & $\begin{array}{l}\text { Obesidad abdominal } \\
\text { OR (IC95\% Li-Ls) }\end{array}$ & $\mathrm{p}$ \\
\hline \multicolumn{5}{|l|}{ Sexo } \\
\hline Mujeres & 1 & & 1 & \\
\hline Hombres & $0,72(0,56-0,91)$ & 0,008 & $0,30(0,23-0,37)$ & $<0,001$ \\
\hline \multicolumn{5}{|l|}{ Edad } \\
\hline 65 a 74 años & 1 & & 1 & \\
\hline 75 a 84 años & $1,10(0,86-1,41)$ & 0,430 & $1,21(0,96-1,53)$ & 0,101 \\
\hline \multicolumn{5}{|l|}{ Nivel educativo } \\
\hline Menos que primarios & 1 & & 1 & \\
\hline Primarios & $0,56(0,42-0,74)$ & $<0,001$ & $0,87(0,67-1,14)$ & 0,309 \\
\hline Secundarios & $0,36(0,23-0,57)$ & $<0,001$ & $0,47(0,32-0,69)$ & $<0,001$ \\
\hline Superiores & $0,30(0,18-0,52)$ & $<0,001$ & $0,38(0,25-0,59)$ & $<0,001$ \\
\hline \multicolumn{5}{|l|}{ Región geográfica } \\
\hline Sur & 1 & & 1 & \\
\hline Norte-oeste & $0,96(0,62-1,49)$ & 0,846 & $0,74(0,49-1,14)$ & 0,173 \\
\hline Norte-este & $0,36(0,20-0,63)$ & $<0,001$ & $0,43(0,26-0,70)$ & 0,001 \\
\hline Centro & $0,66(0,47-0,93)$ & 0,018 & $0,59(0,43-0,82)$ & 0,002 \\
\hline Centro-este & $0,79(0,45-1,38)$ & 0,406 & $0,74(0,43-1,27)$ & 0,275 \\
\hline Este & $0,56(0,40-0,79)$ & 0,001 & $0,63(0,46-0,87)$ & 0,005 \\
\hline \multicolumn{5}{|l|}{ Tamaño de población } \\
\hline Menos de $5.000 \mathrm{~h}$. & 1 & & 1 & \\
\hline Entre 5.000 y $15.000 \mathrm{~h}$. & $1,25(0,84-1,86)$ & 0,264 & $0,53(0,36-0,79)$ & 0,002 \\
\hline Entre 15.000 y $50.000 \mathrm{~h}$. & $0,71(0,47-1,08)$ & 0,110 & $0,47(0,31-0,69)$ & $<0,001$ \\
\hline Entre 50.000 y $200.000 \mathrm{~h}$. & $0,77(0,52-1,13)$ & 0,175 & $0,47(0,32-0,68)$ & $<0,001$ \\
\hline Más de 200.000 h. & $1,03(0,71-1,51)$ & 0,861 & $0,61(0,42-0,89)$ & 0,011 \\
\hline
\end{tabular}

h.: habitantes; IC: intervalo de confianza; Li: límite inferior IC95\%; Ls: límite superior IC95\%; OR: odds ratio.

caso de las personas mayores, la OS se asocia con mayor riesgo de caídas, con el impacto que conllevan sobre la calidad de vida y la fragilidad en las personas de edad avanzada ${ }^{1,25,26}$.

En relación con la distribución geográfica, la prevalencia de obesidad en población $\geq 65$ años en ENPE es mayor en el sur en relación con el norte y el este, patrón similar al descrito en otros grupos de edad y coincidente con datos de otros estudios ${ }^{13,20,21,23,27}$, tanto en adultos más jóvenes como en mayores de 60 años. Se ha postulado que estas diferencias podrían deberse a factores idiosincrásicos de cada comunidad, probablemente relacionados con hábitos dietéticos y estilos de vida ${ }^{22}$, aunque también se ha sugerido la posible influencia del menor nivel socioeconómico en las regiones del $\operatorname{sur}^{20}$.

Entre los predictores sociodemográficos de obesidad y OA se ha observado una relación inversa con el nivel educativo, que también refieren otros estudios en adultos mayores y en otros grupos de $\operatorname{edad}^{13,20,21,23,27}$, y mayor prevalencia en localidades pequeñas, en ámbito rural, en consonancia con hallazgos en otros estudios ${ }^{22}$.

La prevalencia estimada de OA (PC) en este grupo etario $(57,4 \%)$ es algo más elevada que la referida en EXERNET $(55,9 \%)^{21}$, pero con igual distribución en hombres y en mujeres. La prevalencia de OA según este criterio en ENRICA $^{20}$, para este grupo de edad, fue mayor en hombres $(50,9 \%)$ y similar en mujeres, con mayor prevalencia a partir de los 75 años, igual que en ENPE.

En EE. UU., el análisis de datos NHANES 1999-2012, ajustado por edad, estimaba que la prevalencia de OA (PC) ha aumentado en este periodo en casi 8 puntos porcentuales para situarse en el $54,2 \%(2011-2012)^{28}$, a pesar de que la prevalencia de obesidad parece haberse estabilizado. En Reino Unido (38,8\% hombres; 51,2\% mujeres $)^{29}$ y en Portugal $(32,1 \% \text { hombres })^{30}$ se han referido tasas de OA muy por debajo de las estimadas en ENPE, utilizando los mismos criterios, aunque en las mujeres portuguesas de igual edad la prevalencia de OA se sitúa en cifras similares (69,7\%). Datos más recientes en Portugal reportan una prevalencia de OA en población de $65-84$ años, según el índice $C-C$, del $80,2 \%{ }^{19}$, algo por debajo de la estimación en ENPE con este parámetro $(82,2 \%)$.
Estos datos reflejan una situación y una tendencia preocupante entre los adultos mayores españoles, ya que no solo existe una mayor prevalencia de sobrepeso y obesidad, sino también de OA, lo que acentúa la necesidad de implementar urgentemente medidas para frenar o revertir dicha tendencia.

Existe evidencia de que los adultos mayores con obesidad y sarcopenia tienen peor capacidad funcional y parece que ambos problemas actúan de forma sinérgica, aumentando el riesgo de discapacidad $^{25}$. En ENPE se ha estimado la prevalencia de OS en un $10,3 \%$ en hombres y un $10,9 \%$ en mujeres, cifras inferiores a las referidas en EXERNET $(15 \%)^{21}$ y en la cohorte ENRICA-Senior $(17,2 \%)^{26}$, aunque en ambos estudios se estimó la masa muscular mediante impedanciometría. Son limitados los estudios poblacionales con estimaciones de prevalencia de OS y es difícil la comparación porque no existe consenso sobre su definición ${ }^{12}$.

La obesidad, OA y OS aumentan el riesgo de comorbilidades asociadas con mayor riesgo de discapacidad, peor calidad de vida y mortalidad $^{25}$. En este análisis se ha observado que las tres condiciones se asocian de forma independiente con mayor probabilidad de hipercolesterolemia e HTA y la OS, además, con diabetes, en consonancia con los hallazgos en otros estudios ${ }^{22,27}$.

Entre las fortalezas del estudio ENPE cabe destacar que se ha realizado en una muestra aleatoria representativa de la población española no institucionalizada, considerando la distribución geográfica. El cuidado diseño del protocolo de estudio, adiestramiento de encuestadores y control de calidad durante el trabajo de campo proporcionan estimaciones actualizadas de prevalencia de obesidad, OA según distintos indicadores y OS en población de 65 y más años. Como limitaciones, cabe mencionar que la información sobre problemas de salud de los pacientes se ha considerado a partir de información reportada por los encuestados y no mediante estimaciones objetivas $\mathrm{y}$, por otro lado, el diseño transversal del estudio no permite establecer relaciones de causalidad.

Los resultados de este estudio ponen de manifiesto tasas de prevalencia de obesidad y especialmente de OA muy elevadas en la 

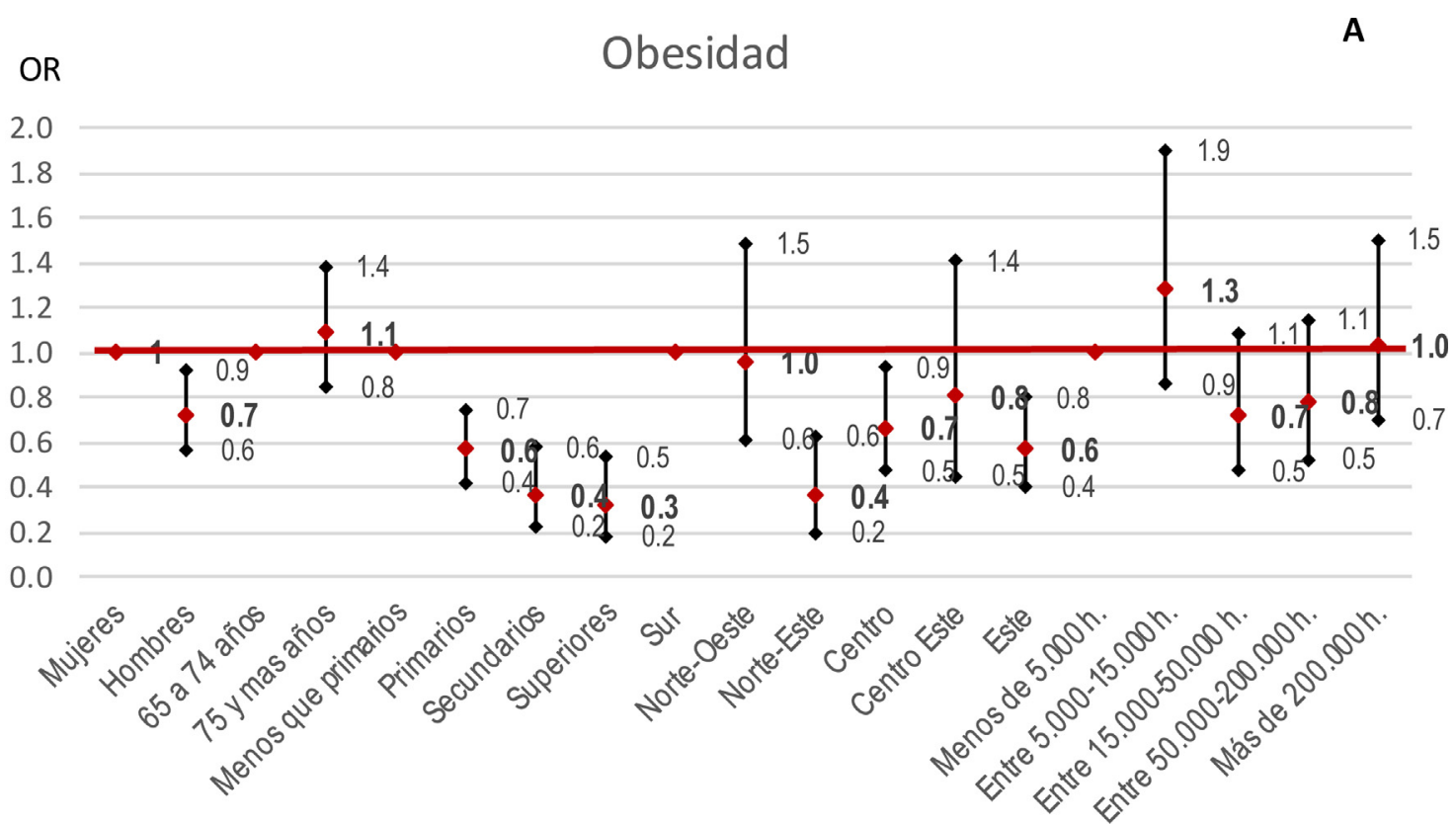

$\overline{\text { Sexo }} \overline{\text { Edad }}$

Nivel educativo

Región geográfica

Tamaño de hábitat

OR

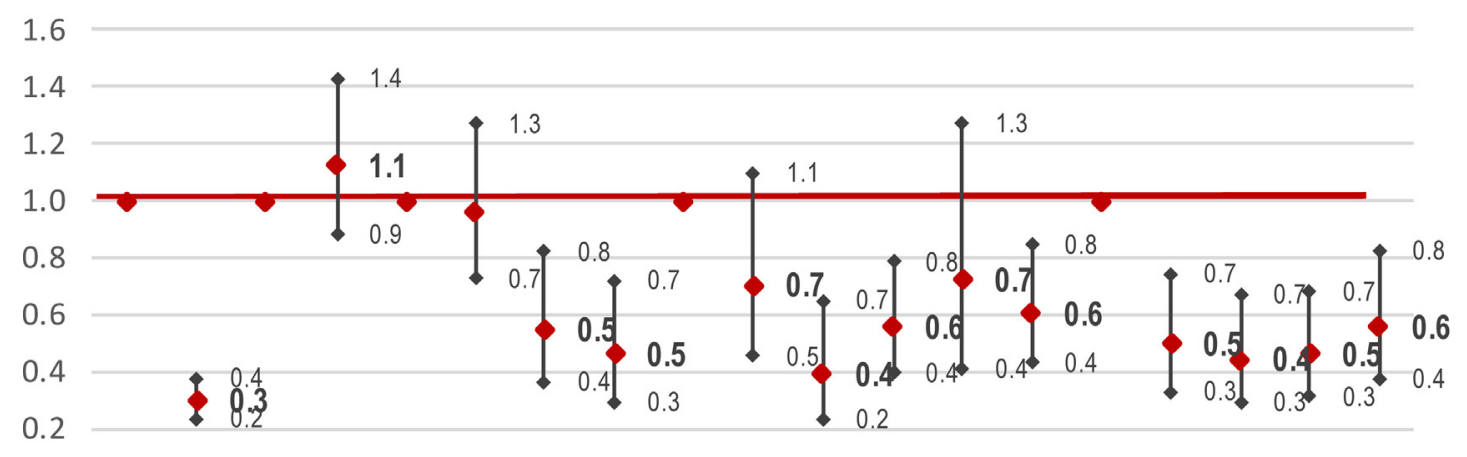

0.0

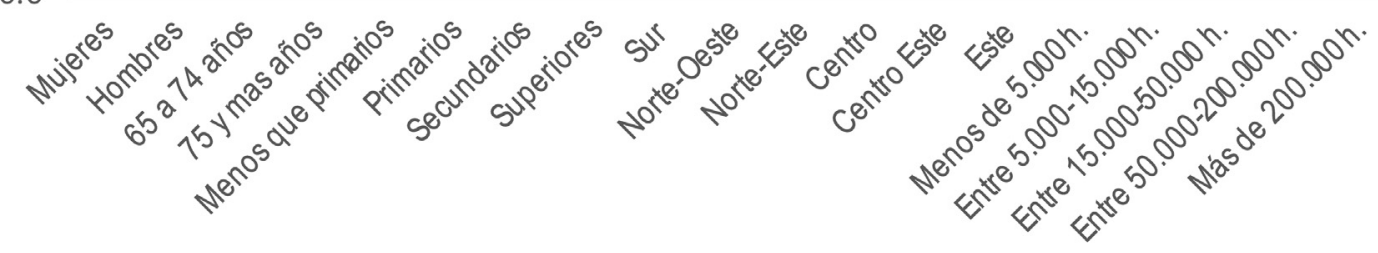

Sexo Edad Nivel educativo

Región geográfica

Tamaño de hábitat

Figura 1. Condicionantes sociodemográficos de la obesidad (A) y obesidad abdominal (B) en adultos $\geq 65$ años de edad.

Análisis de regresión logística ajustado por edad y sexo. Los rombos representan OR de prevalencia ajustadas y las barras el intervalo de confianza al $95 \%$. Obesidad abdominal según perímetro de cintura $>102 \mathrm{~cm}$ en varones $\mathrm{y}>88 \mathrm{~cm}$ en mujeres.

población de 65 y más años. El aumento en la esperanza de vida hace necesario conseguir mejorar la calidad de vida en la edad avanzada. Para ello, promover la adecuación de los hábitos alimentarios, la práctica de actividad física y reducir el sedentarismo son claves para la prevención del problema desde edades tempranas, considerando especialmente los grupos socioeconómicos menos favorecidos y los entornos urbanos en las localidades de menor tamaño para reforzar los recursos e iniciativas de promoción de la salud en todos los grupos de edad. También en la edad adulta, con particular atención y apoyo a las personas mayores.

En conclusión, la prevalencia de obesidad en población de 65 y más años en España es alta, mayor en mujeres, en personas con menor nivel educativo y en la región sur. Es especialmente preocupante la alta prevalencia de OA en este grupo de edad, sobre todo en las mujeres, por su asociación con complicaciones cardiovasculares, metabólicas, fragilidad general y deterioro de la calidad de vida. 


\section{Hipercolesterolemia}

A

$\mathrm{OR}$

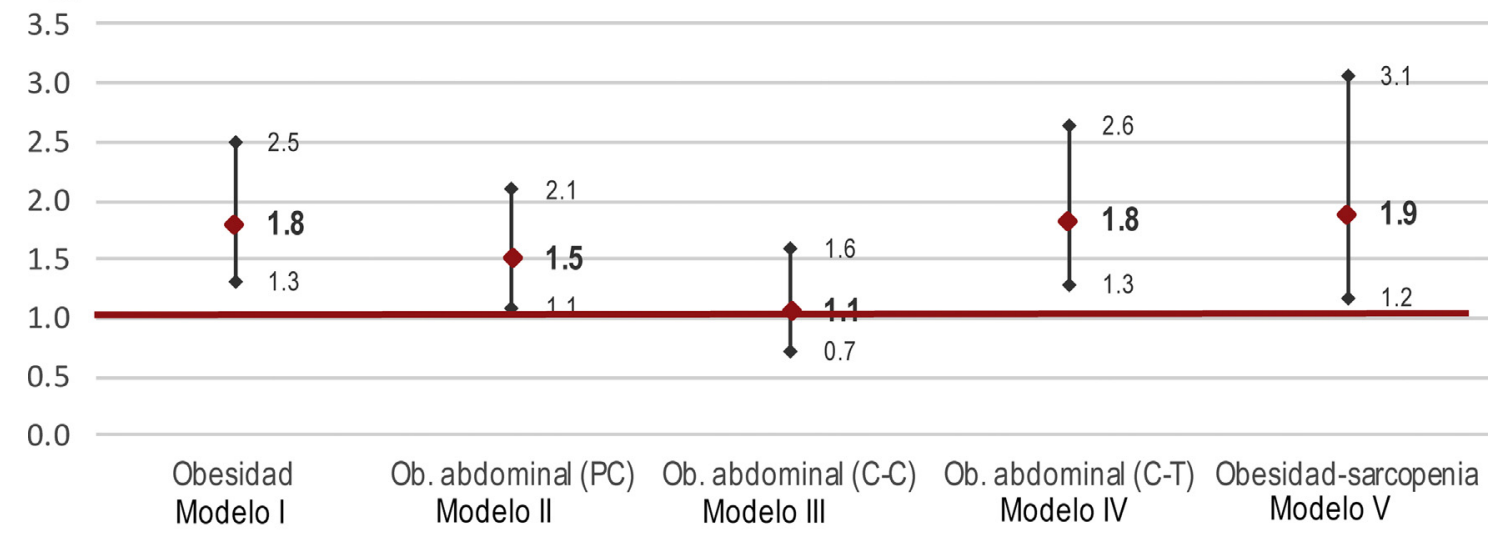

OR

Hipertensión arterial

B

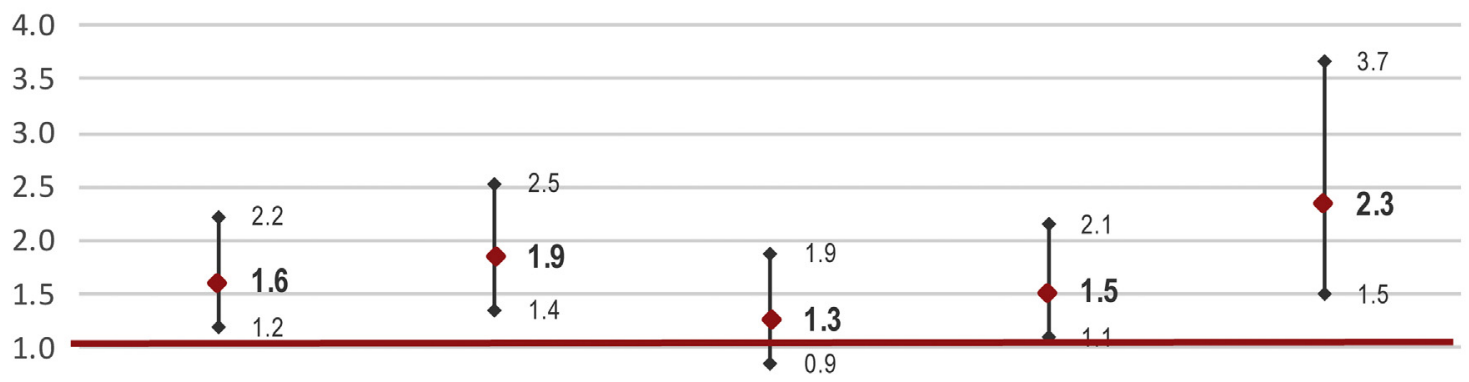

0.5

0.0

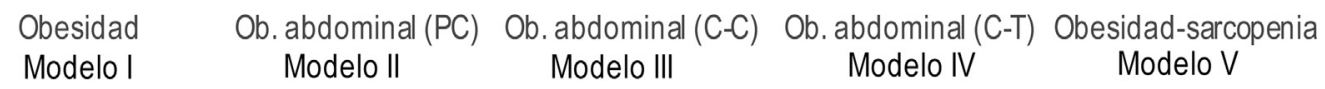

OR

Diabetes

C

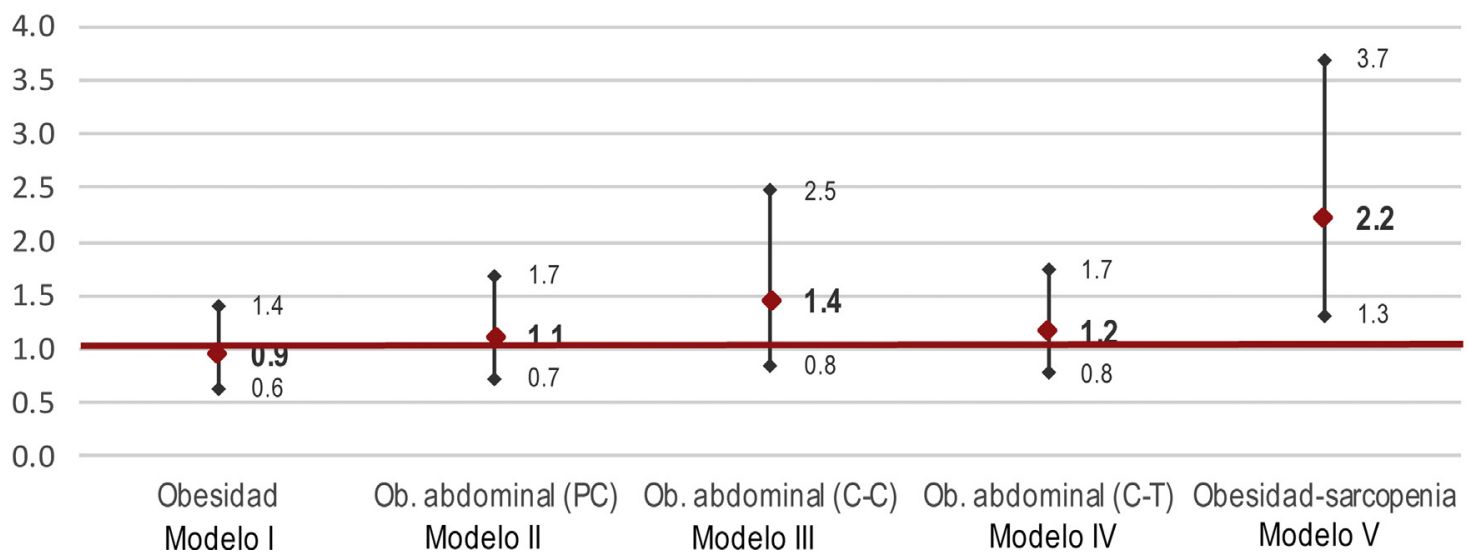

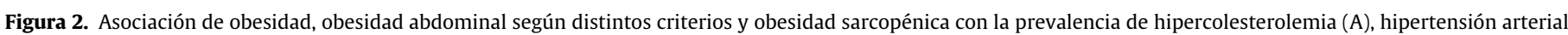
(B) y diabetes (C) en adultos $\geq 65$ años de edad.

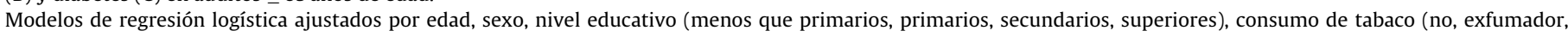

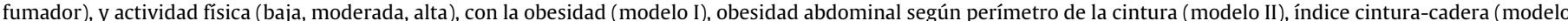

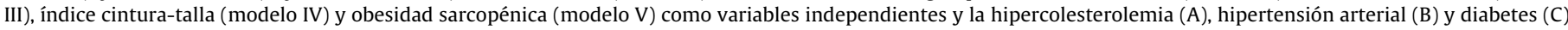
como variables dependientes. Los rombos representan OR de prevalencia ajustadas y las barras el intervalo de confianza al 95\%.

Ob abdominal (PC): obesidad abdominal definida según el valor del perímetro de cintura.

Ob abdominal (C-C): obesidad abdominal definida según el índice cintura-cadera.

Ob abdominal (C-T): obesidad abdominal definida según el índice cintura-talla.

Cómo citar este artículo: Pérez-Rodrigo C, et al. Prevalencia de obesidad y obesidad abdominal en la población española de 65 y más

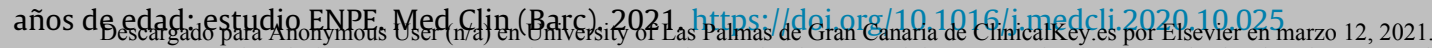




\section{Financiación}

El estudio ENPE fue financiado por la Fundación Eroski a través de un acuerdo con SPRIM y la Sociedad Española de Nutrición Comunitaria (SENC). El patrocinador no ha intervenido en el diseño del estudio, la recogida de datos, el análisis o la interpretación de los resultados, la redacción del manuscrito o la decisión de publicar los resultados.

\section{Conflicto de intereses}

Los autores no tienen conflicto de interés que declarar en relación con este trabajo.

\section{Agradecimientos}

Los autores agradecen el soporte técnico y logístico de la empresa SIGMA DOS, el apoyo técnico brindado por SPRIM-España, el patrocinio de la Fundación Eroski y, de manera especial, la labor desarrollada por los profesionales del trabajo de campo y la generosidad de las personas que accedieron a participar en el estudio.

\section{Bibliografía}

1. Batsis JA, Zagaria AB. Addressing obesity in aging patients. Med Clin North Am. 2018;102:65-85.

2. Blüher M. Obesity: global epidemiology and pathogenesis. Nat Rev Endocrinol. 2019;15:288-98

3. NCD Risk Factor Collaboration (NCD-RisC). Trends in adult body-mass index in 200 countries from 1975 to 2014: a pooled analysis of 1698 population-based measurement studies with 19.2 million participants. Lancet. 2016;387:1377-96.

4. Marques A, Peralta M, Naia A, Loureiro N, de Matos MG. Prevalence of adult overweight and obesity in 20 European countries, 2014. Eur J Public Health. 2018:28:295-300.

5. Peralta M, Ramos M, Lipert A, Martins J, Marques A. Prevalence and trends of overweight and obesity in older adults from 10 European countries from 2005 to 2013. Scand J Public Health. 2018;46:522-9.

6. Basterra-Gortari FJ, Bes-Rastrollo M, Ruiz-Canela M, Gea A, Martinez-Gonzalez MÁ. Prevalence of obesity and diabetes in Spanish adults 1987-2012. Med Clin (Barc). 2017;148:250-6.

7. Hernáez Á, Zomeño MD, Dégano IR, Pérez-Fernández S, Goday A, Vila J, et al. Excess weight in Spain: Current situation projections for 2030, and estimated direct extra cost for the Spanish Health System. Rev Esp Cardiol. 2019;72:916-24, http://dx.doi.org/10.1016/j.rec.2018.10.010.

8. The Global BMI Mortality Collaboration. Body-mass index and all-cause mortality: individual participant-data meta-analysis of 239 prospective studies in four continents. Lancet. 2016;388:776-86.

9. WHO. Waist circumference and waist-hip ratio: report of a WHO Expert Consultation. Geneva, 8-11 December 2008. Ginebra: WHO; 2011 [consultado 15 Jun 2020). Disponible en: http://www.who.int/ nutrition/publications/obesity/WHO_report_waistcircumference_and_waisthip _ratio/en/

10. Ashwell M, Gibson S. Waist-to-height ratio as an indicator of 'early health risk': simpler and more predictive than using a 'matrix' based on BMI and waist circumference. BMJ Open. 2016;63:e010159.

11. Corrêa MM, Thumé E, De Oliveira ER, Tomasi E. Performance of the waist-toheight ratio in identifying obesity and predicting non-communicable diseases in the elderly population: A systematic literature review. Arch Gerontol Geriatr. 2016;65:174-82.

12. Cruz-Jentoft AJ, Bahat G, Bauer J, Boirie Y, Bruyère O, Cederholm T, et al. Sarcopenia: revised European consensus on definition and diagnosis. Age Ageing. 2019;48:16-31.

13. Aranceta-Bartrina J, Pérez-Rodrigo C, Alberdi-Aresti G, Ramos-Carrera N, LázaroMasedo S. Prevalencia de obesidad general y obesidad abdominal en la población adulta española (25-64 años) 2014-2015: estudio ENPE. Rev Esp Cardiol. 2016;69:579-87.

14. Stewart A, Marfell-Jones M. International Society for Advancement of Kinanthropometry. International standards for anthropometric assessment. Lower Hutt, New Zealand: International Society for the Advancement of Kinanthropometry; 2011. p. 83-5.

15. Tomioka K, Iwamoto J, Saeki K, Okamoto N. Reliability and validity of the International Physical Activity Questionnaire (IPAQ) in elderly adults: The Fujiwarakyo Study. J Epidemiol. 2011;21:459-65.

16. Mathus-Vliegen EM, Obesity Management Task Force of the European Association for the Study of Obesity. Prevalence, pathophysiology, health consequences and treatment options of obesity in the elderly: a guideline. Obes Facts. 2012;5:460-83

17. Flegal KM, Kruszon-Moran D, Carroll MD, Fryar CD, Ogden CL. Trends in obesity among adults in the United States, 2005 to 2014. JAMA. 2016;315:2284-91.

18. Lopez-Ortega M, Arroyo P. Anthropometric characteristics and body composition in Mexican older adults: Age and sex differences. Br J Nutr. 2016;115:490-9.

19. Oliveira A, Araújo J, Severo M, Correia D, Ramos E, Torres D, et al., by the IAN-AF Consortium. Prevalence of general and abdominal obesity in Portugal: comprehensive results from the National Food, nutrition and physical activity survey 2015-2016. BMC Public Health. 2018;18:614.

20. Gutierrez-Fisac JL, Guallar-Castillon P, Leon-Munoz LM, Graciani A, Banegas JR, Rodriguez-Artalejo F. Prevalence of general and abdominal obesity in the adult population of Spain, 2008-2010: The ENRICA study. Obes Rev. 2012;13:388-92.

21. Gomez-Cabello A, Pedrero-Chamizo R, Olivares PR, Luzardo L, Juez-Bengoechea A, Mata E, et al. Prevalence of overweight and obesity in non-institutionalized people aged 65 or over from Spain: The elderly EXERNET multi-centre study. Obes Rev. 2011;12:583-92.

22. Cea-Calvo L, Moreno B, Monereo S, Gil-Guillén V, Lozano JV, Martí-Canales JC, et al. Prevalence and related factors of overweight and obesity in Spanish population aged 60 years-old or older. the PREV-ICTUS study. Med Clin (Barc). 2008;131:205-10.

23. Aranceta-Bartrina J, Serra-Majem L, Foz-Sala M, Moreno-Esteban B. Prevalencia de obesidad en España. Med Clin(Barc). 2005;125:460-6.

24. Gutierrez-Fisac JL, Lopez E, Banegas JR, Graciani A, Rodriguez-Artalejo F. Prevalence of overweight and obesity in elderly people in Spain. Obes Res. 2004;12:710-5.

25. Batsis JA, Mackenzie TA, Jones JD, Lopez-Jimenez F, Bartels SJ. Sarcopenia, sarcopenic obesity and inflammation: Results from the 1999-2004 National Health and Nutrition Examination Survey. Clin Nutr. 2016;35:1472-83.

26. Moreno-Franco B, Pérez-Tasigchana RF, Lopez-Garcia E, Laclaustra M, Gutierrez-Fisac JL, Rodríguez-Artalejo F, et al. Socioeconomic determinants of sarcopenic obesity and frail obesity in community-dwelling older adults: The Seniors-ENRICA Study. Sci Rep. 2018;8:10760, http://dx.doi.org/10.1038/s41598-018-28982-x.

27. Gabriel R, Alonso M, Segura A Tormo MJ, Artigao LM, Banegas JR, et al. Prevalence, geographic distribution and geographic variability of major cardiovascular risk factors in Spain. Pooled analysis of data from population-based epidemiological studies: The ERICE study. Rev Esp Cardiol. 2008;61:1030-40.

28. Ford ES, Maynard LM, Li C. Trends in mean waist circumference and abdominal obesity among US adults, 1999-2012. JAMA. 2014;312:1151-3.

29. Morrell J, Fox KA. Prevalence of abdominal obesity in primary care: The IDEA UK study. Int J Clin Pract. 2009:63:1301-7.

30. Sardinha LB, Santos DA, Silva AM, Coelho-e-Silva MJ, Raimundo AM, Moreira H, et al. Prevalence of overweight, obesity, and abdominal obesity in a representative sample of Portuguese adults. PLoS One. 2012;7:e47883. 